\title{
The Argonautes
}

\author{
L. JOSHUA-ToR \\ Cold Spring Harbor Laboratory, Cold Spring Harbor, New York 11724
}

\begin{abstract}
RNA interference (RNAi) has been greatly exploited in recent years as an increasingly effective tool to study gene function by gene silencing. The introduction of exogenous double-stranded RNA (dsRNA) into a cell can trigger this gene silencing process. An RNase III family enzyme, Dicer, initiates silencing by releasing approximately 20 base duplexes, with 2nucleotide 3' overhangs called siRNAs. The RNAi pathway also mediates the function of endogenous, noncoding regulatory RNAs called miRNAs. Both miRNAs and siRNAs guide substrate selection by similar if not identical effector complexes called RISCs. These contain single-stranded versions of the small RNA and additional protein components. Of those, the signature element, at the heart of all RISCs, is a member of the Argonaute family of proteins. Our structural and biochemical studies on Argonaute identified this protein as Slicer, the enzyme in RISC that cleaves the mRNA as directed by the siRNA. The role of the Argonautes as Slicers and non-Slicers is discussed.
\end{abstract}

RNAi has made an enormous impact on biology in a very short period of time. It became an extraordinarily useful and simple tool for gene silencing, even as its mechanism was gradually unraveling. Deciphering the mechanism of RNAi-related pathways, including both transcriptional and posttranscriptional gene silencing, has benefited from an incredible marriage of genetics, biochemistry, molecular biology, and, of course, structural biology.

\section{RNAI EFFECTOR COMPLEXES}

The various pathways of RNAi can be broken down into the initiation steps in which the dsRNA triggers are processed into siRNAs or miRNAs, and the effector steps in which silencing is realized. This is, of course, an oversimplified view of these related pathways; however, in each case, the effector step involves an effector complex called the RNA-induced silencing complex (RISC) (Hammond et al. 2000). RISCs of varying compositions and sizes have been purified from both human and fly cells (Hammond et al. 2001; Nykanen et al. 2001; Caudy et al. 2002, 2003; Ishizuka et al. 2002; Martinez et al. 2002). However, the minimal defining features of RISC include the presence of an Argonaute family member and the guide strand of a short RNA. The short RNA (si- or miRNA) then acts to guide the RISC to its target through base complementarity. The best-characterized pathway, and the one that is predominantly used for gene knockdown technology, is a posttranscriptional silencing (PTGS) pathway called Slicing. Here the RISC is targeted to the mRNA and produces an endonucleolytic cut in the mRNA target, thus preventing gene expression from proceeding (for a recent review, see Zamore and Haley 2005). This cut is made 10 bases from where the $5^{\prime}$ end of the guide siRNA hybridizes to the target mRNA (Elbashir et al. 2001a, b). Other RNAi silencing pathways such as translational inhibition and transcriptional gene silencing (TGS) are also mediated through RISCs. In the fission yeast, Schizosaccharomyces pombe, there is a specialized RISC, called RITS, that is composed of Ago1, the sole
Argonaute protein in $S$. pombe; Tas3; and a chromodomain protein, Chp1 (Verdel et al. 2004). This complex is involved in regulation of silencing of centromeric repeats and other heterochromatic loci. The crystal structure of Argonaute from Pyrococcus furiosus (PfAgo), described below, revealed an RNase H-like PIWI domain capable of RNA cleavage (Song et al. 2004). This established Argonaute as the catalytic core of the effector complex, RISC (Liu et al. 2004; Song et al. 2004).

\section{ARGONAUTE-THE CATALYTIC CORE OF RISC}

As mentioned above, the Argonaute proteins are at the heart of RISC. They have two characteristic domains: the PAZ domain, which is shared with another key RNAi enzyme, Dicer; and a unique domain called the PIWI domain. Both PAZ and PIWI domains appear to be unique to proteins involved in RNAi-type processes. The PAZ domain is approximately 140 amino acids in length and is named after three proteins-Piwi, Argonaute, and Zwille. Three PAZ domain structures were determined and published concurrently: the NMR structure of the PAZ domain of Drosophila melanogaster Agol by the Zhou group (Yan et al. 2003), the NMR structure of the PAZ domain of $D$. melanogaster Ago2 by the Sattler group (Lingel et al. 2003), and our crystal structure of the same PAZ domain $D$. melanogaster Ago2 (Song et al. 2003). The structures are all similar to each other and contain two subdomains with a prominent cleft between. The larger subdomain has a fold that is reminiscent of an OB-fold domain, although with slightly different topology, and the second subdomain, called by some "the appendage," is composed of a $\beta$-hairpin followed by an $\alpha$-helix. OB fold stands for oligonucleotide-oligosaccharide binding, since a majority of OB-fold proteins bind single-stranded oligonucleotides or oligosaccharides. This suggested a role for the PAZ domain in nucleic acid binding. A series of crosslinking and mutagenesis experiments showed that the siRNAs bind to the PAZ intersubdomain cleft through the 2-nucleotide over- 
hang at their $3^{\prime}$ end (Song et al. 2003). This mode of PAZ-siRNA recognition was confirmed shortly after by structures of the PAZ in complex with nucleic acids (Lingel et al. 2004; Ma et al. 2004) that also showed binding to be sequence independent. Highly conserved aromatic residues - two tyrosines and a histidine - contact the phosphate between the two bases in the overhang.

The structure of the full-length Argonaute from $P$. furiosus (PfAgo) was critical in defining the function of Argonaute (Song et al. 2004). It showed the Argonautes to be composed of four domains: the amino-terminal, PAZ, middle, and PIWI domains. Overall, the protein has a crescent-shaped base comprising the amino, middle, and PIWI domains. The region following the amino-terminal domain forms a "stalk" that holds the PAZ domain above the rest of the structure (Fig. 1). An interdomain connector cradles the four domains of the molecule. This architecture forms a large, positively charged groove between the PAZ domain and the crescent base, and a smaller one between the amino and PIWI domains.

The PAZ domain of PfAgo is very similar in structure to all the other PAZ domain structures that were determined previously, despite a less than $6 \%$ sequence identity. Importantly, the side chains of the aromatic residues in the PAZ intersubdomain cleft that were shown to bind the $3^{\prime}$ end of the siRNA fall in the same positions in space. Y212, Y216, H217, and Y190 of PfAgo are equivalent to residues Y309, Y314, H269, and Y277 of human Ago1 (hAgo1) (Ma et al. 2004; Song et al. 2004). However, in the case of the histidine of PfAgo, it comes from a very different location on the backbone than the equivalent one from hAgol. Indeed, the sequences of the PfAgo and human Agol can only be aligned using the two structures. The middle

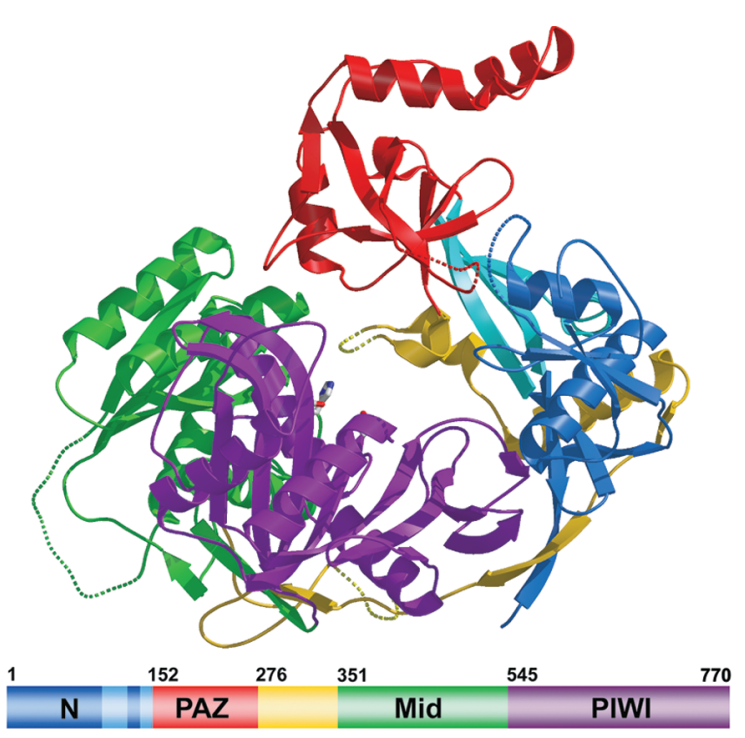

Figure 1. Crystal structure of $P$. furiosus Argonaute. Stereo ribbon representation of Argonaute with the amino-terminal domain shown in blue, the "stalk" in light blue, the PAZ domain in red, the middle domain in green, the PIWI domain in purple, and the interdomain connector in yellow. The active-site aspartates and histidine residues are drawn in stick representation. Disordered loops are drawn as dotted lines. Shown below is a schematic diagram of the domain borders.

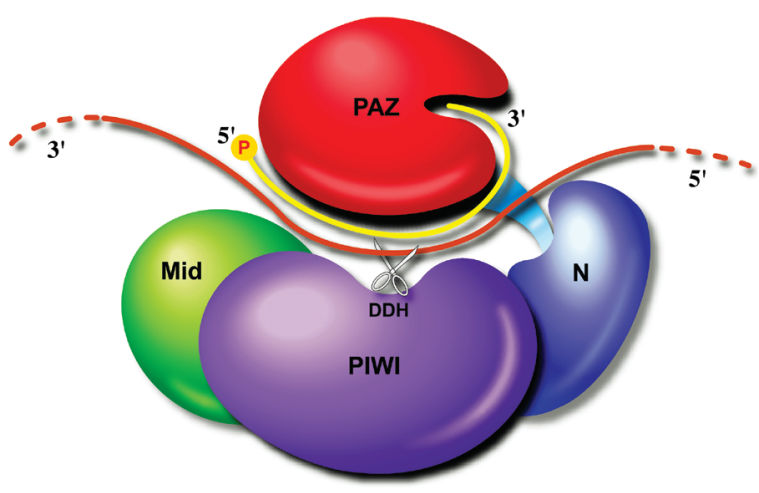

Figure 2. Schematic depiction of the model for siRNA-guided mRNA cleavage. The domains are colored as in Fig. 1. The siRNA, shown in yellow, binds with its $3^{\prime}$ end in the PAZ cleft. Its $5^{\prime}$ phosphate is important for enzyme stability and fidelity. The mRNA is depicted in brown, comes in between the aminoterminal and PAZ domains and out between the PAZ and middle domain. The active site in the PIWI domain, depicted as scissors, with its DDH motif, cleaves the mRNA opposite the middle of the siRNA guide.

domain is similar in structure to sugar-binding proteins such as the sugar-binding domain of Lac repressor.

The big surprise came upon examination of the PIWI domain. It is at the carboxyl terminus of Argonaute and located across the large groove from the PAZ domain. Not much was known about it from sequence alone, and it appeared to reside only in Argonaute proteins. The crystal structure revealed that it clearly belongs to the RNase $\mathrm{H}$ family of enzymes (Song et al. 2004). Apart from the RNase $\mathrm{H}$ fold at its core, it also has the two highly conserved aspartates always present on adjacent $\beta$-strands in these types of enzymes. The sequence of the PIWI domain of PfAgo aligns well with other members of the Argonaute family. Modeling of siRNA and target (mRNA) binding starting from the PAZ $3^{\prime}$ end binding site revealed that the large groove could accommodate the duplex positioning the predicted scissile phosphate at the active site near the two aspartates. Directed by the structure, mutation of each of these aspartates that are conserved in human Argonaute2 eliminates Slicer activity (Liu et al. 2004; Rivas et al. 2005). These findings identified Argonaute as being the long-sought catalytic enzyme of RISC, dubbed Slicer, that slices the mRNA substrate guided by the siRNA (Fig. 2). Moreover, the dependence of RISC Slicer activity on the presence of $\mathrm{Mg}^{2+}$ ions and the generation of similar products to RNase $\mathrm{H}$ family enzymes further underscores this conclusion.

The crystal structure of AfPIWI (Parker et al. 2004), which possesses both middle and PIWI domains but lacks the amino-terminal and PAZ domains, also demonstrated the similarity to Lac repressor and RNase H. Because this protein does not contain the catalytic aspartates, it does not bind a divalent metal ion in the active site. However, a $\mathrm{Cd}^{2+}$ ion is bound to the carboxy-terminal carboxylate of the protein at a conserved region of the protein. The carboxyl terminus of both AfPiwi and PfAgo lies at the interface between the mid and Piwi domains. Structures of a complex of this protein with dsRNA were later deter- 
mined that suggest a mode of recognition for the $5^{\prime}$ phosphate of the guide RNA (Ma et al. 2005; Parker et al. 2005). The $5^{\prime}$ nucleotide of the siRNA is unpaired, and the base and phosphate interact with residues of the middle domain. This is consistent with the observation that only nucleotides 2-8 of the guide RNA (the "seed" sequence) play a role in target recognition and specificity (Lewis et al. 2003; Stark et al. 2003; Doench and Sharp 2004). Coordination of the $5^{\prime}$ phosphate is also dependent on the $\mathrm{Cd}^{2+}$ metal ion. However, the metal ion is not observed in the apo and manganese-soaked crystals of PfAgo (Rivas et al. 2005) and may require the presence of the guide strand for coordination if the mode of siRNA binding is similar between these proteins.

More recently, the structure of a eubacterial Argonaute protein from Aquifex aeolicus (AaAgo) was determined and shows identical domain architecture as PfAgo (Yuan et al. 2005). However, the relative orientation of the PAZ domain is quite different. Interestingly, this protein was shown to have RNase-H-like activity, whereby it shows preference for a DNA/RNA hybrid as a substrate.

\section{ACTIVE SITE OF SLICER}

A combination of crystallographic and mutational analyses showed that Argonaute contains two aspartates and a histidine at its active site in a conserved "DDH" motif (Liu et al. 2004; Song et al. 2004; Rivas et al. 2005). A crystal structure of PfAgo soaked with manganese chloride showed that the $\mathrm{Mn}^{2+}$ ion bound these three residues (Rivas et al. 2005). This is equivalent to metal ion binding in other RNase $\mathrm{H}$ family enzyme structures lacking substrate. A second metal ion is present in structures of Tn5 transposase (Lovell et al. 2002) and RNase H (Nowotny et al. 2005) with bound substrate. It is reasonable to suggest that Argonaute uses a similar two-metal-ion mechanism for catalysis.

The two aspartates appear to be critical to convey slicer activity; however; it appears that the histidine might be replaced by another aspartate, a glutamate, or even a lysine, resulting in a more relaxed active site requirement of $\mathrm{DD}(\mathrm{H} / \mathrm{D} / \mathrm{E} / \mathrm{K})$ (Table 1). Of the four immunopurified Argonautes tested (hAgo1-4), the only one with slicer activity is hAgo2 (Liu et al. 2004; Meister et al. 2004). hAgol has an arginine in place of the histidine, and hAgo4 has a glycine in place of one of the aspartates. Three of the four D. melanogaster Argonautes have been tested for activity-DmAgol (Miyoshi et al. 2005) and DmAgo2 (Rand et al. 2005) with DDH, and DmPiwi (Saito et al. 2006) with DDK, and all have slicer activity. Arabidopsis has 10 family members with either DDH or DDD as active-site residues, although only AtAgol and AtAgo4 have been shown to exhibit slicer activity (Baumberger and Baulcombe 2005; Qi et al. 2005, 2006). Caenorhabditis elegans has as many as 24 Argonaute proteins. Although none of these, to our knowledge, has been shown in vitro to have slicer activity, some, such as CeRDE-1, function via slicing (Tabara et al. 1999). The sole Argonaute in $S$. pombe, SpAgo1, has a complete $\mathrm{DDH}$, and we have now shown that it is active for slicing in vitro (Irvine et al. 2006).
The presence of the catalytic residues is necessary for slicer activity, but it is not the whole story, hAgo3 being a case in point. hAgo 3 has a complete DDH motif similar to hAgo2, but the protein immunopurifed from 293 or HeLa cells does not appear to have slicer activity, even though it is able to bind siRNA (Liu et al. 2004; Meister et al. 2004).

Previously, a phylogenic analysis of Argonaute proteins showed that they can be divided into two classes - the Argonaute-like Argonautes that are similar to AtAgo1 and the Piwi-like Argonautes that are similar to DmPiwi. We performed a new analysis including more Argonaute proteins from Homo sapiens, Arabidopsis thaliana, C. elegans, D. melanogaster, and S. pombe (Yigit et al. 2006). This suggests the existence of a third clade which we termed the Group III Argonautes (Table 1). Interestingly, this clade is worm-specific and predominantly contains non-Slicer-like Argonautes, meaning they do not have an intact catalytic motif. In contrast, the Ago-like and Piwi-like clades contain a high proportion of proteins from all species that are either Slicers, which have been shown to have slicer activity, or are Slicer-like, those which contain the three catalytic residues. Thus, we postulate that the worm has evolved a large set of non-Slicer-like Argonautes. Inclusion of 21 Argonautes from Caenorhabditis briggsae in a similar analysis showed that $C$. briggsae also possesses Group III Argonautes. Thus, the divergence of the Group III Argonautes arose prior to the $C$. elegans-C. briggsae split. Interestingly, CeRde-1, CePpw-1, and CePpw-2 also cluster in this clade. CeRde-1 was the first Argonaute family member to be genetically linked to silencing (Tabara et al. 1999), as CeRde-1 mutants have a penetrant loss of RNAi. CePpw-1 and CePpw-2 appear to be involved in RNAi in the germ line of C. elegans (Tijsterman et al. 2002; Vastenhouw et al. 2003).

\section{TGS AND SLICING}

Slicing of the substrate mRNA has long been established for PTGS. Recently, we have shown that SpAgo1 is an active Slicer that is required for TGS in $S$. pombe (Irvine et al. 2006). Mutation of active-site aspartate residues of SpAgo1 results in a defective enzyme in vitro. In vivo, this results in a derepression of silencing at heterochromatic repeats and a reduction in histone $\mathrm{H} 3$ lysine 9 dimethylation as well as an increase in histone $\mathrm{H} 3$ lysine 4 methylation. SpAgol levels at these repeats were observed at similar levels for the Slicer mutant strains, although RNA-dependent RNA polymerase (RdRP) levels were decreased, implicating slicing and the production of a free $3^{\prime} \mathrm{OH}$ as a requirement for recruitment of the RdRP complex (RDRC) to target RdRP activity, which in turn would initiate spreading.

\section{MINIMAL RISC AND THE SLICING CYCLE}

Because hAgo2 and hAgo3 were immunopurified from RNAi-competent cells (Liu et al. 2004; Meister et al. 2004), the differences in activity between them may have been due to a difference in purity between the two preparations, giving hAgo 2 its activity. To eliminate this trivial 
Table 1. Catalytic Residues of Argonautes from H. sapiens (Hs), A. thaliana (At), D. melanogaster (Dm), S. pombe (Sp), and C. elegans (Ce).

\begin{tabular}{|rllllllllll|}
\hline HsAgo1 & VIFLGA & D & V & IFYR & D & GVP & IPAPAYYA & R & LVAF & Ago \\
HsAgo2 & VIFLGA & D & V & IFYR & D & GVS & IPAPAYYA & H & LVAF & Ago \\
HsAg03 & VIFLGA & D & V & IFYR & D & GVS & IPAPAYYA & H & LVAF & Ago \\
HsAg04 & VIFLGA & D & V & IYYR & G & GVS & IPAPAYYA & R & LVAF & Ago \\
HsHIWI / PIWIL1 & VMIVGI & D & C & IVYR & D & GVG & VPAPCQYA & H & KLAF & PIWI \\
HsHILI / PIWIL2 & LMVIGM & D & V & VVYR & D & GVS & VPAPCKYA & H & KLAF & PIWI \\
HsPIWIL3 & TMFVGI & D & C & IVYR & D & GVG & VPAPCHYA & H & KLAY & PIWI \\
HsHIWI2 / PIWIL4 & LMVVGI & D & V & IVYR & A & GVG & VPAPCQYA & H & KLTF & PIWI \\
\hline
\end{tabular}

\begin{tabular}{|lllllllllll|}
\hline AtAg01 & TIIFGA & D & V & IFYR & D & GVS & IVPPAYYA & H & LAAF & Ago \\
AtAgo2 & VMFIGA & D & V & VIFR & D & GVS & LVPPVYYA & D & MVAF & Ago \\
AtAgo3 & VMFIGA & D & V & VIFR & D & GVS & LVPPVSA & D & KAAS & Ago \\
AtAg04 & TIILGM & D & V & IIFR & D & GVS & VVAPICYA & H & LAAA & Ago \\
AtAg05 & TIIMGA & D & V & IFYR & D & GVS & IVPPAYYA & H & LAAF & Ago \\
AtAg06 & TLILGM & D & V & IIFR & D & GVS & SVAPVRYA & H & LAAA & Ago \\
AtAgo7 & VIFMGA & D & V & IFFR & D & GVS & IVPPAYYA & H & LAAY & AgO \\
AtAg08 & TIIIGM & D & V & IFYR & D & GVS & VVAPVCYA & H & LAAA & AgO \\
AtAgo9 & TIIVGM & D & V & IIFR & D & GVS & VVAPVCYA & H & LAAA & AgO \\
AtZII/Pnh & TIIFGA & D & V & IFYR & D & GVS & IVPPAYYA & H & LAAF & AgO \\
\hline
\end{tabular}

\begin{tabular}{|c|c|c|c|c|c|c|c|c|c|c|}
\hline SpAgo1 & TLILGG & D & $\overline{\mathrm{V}}$ & $\overline{\text { IYFR }}$ & D & $\overline{G T S}$ & LVPPVYYA & $\mathrm{H}$ & LVSN & Ago \\
\hline CeZK757.3 & TMVVGI & $\bar{D}$ & $\overline{\mathrm{V}}$ & $\overline{\text { IVYR }}$ & $\bar{D}$ & $\overline{G V S}$ & IPTPVYYA & $\overline{\mathrm{D}}$ & LVAT & $\overline{A g o}$ \\
\hline CeT22B3.2 & TMVVGI & D & V & IVYR & D & GVS & IPTPVYYA & $\mathrm{D}$ & LVAT & Ago \\
\hline CeT23D8.7 & VLFIGC & $\mathrm{H}$ & $\mathrm{L}$ & IIYR & A & GIA & IPSPVYYA & K & LVAQ & Ago \\
\hline CeAlg-1 & VIFFGC & D & I & VVYR & D & GVS & IPAPAYYA & H & LVAF & Ago \\
\hline CeAlg-2 & VIFLGC & D & I & VVYR & D & GVS & IPAPAYYA & H & LVAF & Ago \\
\hline CeR09A1.1 & TLVLGI & D & $\overline{\mathrm{V}}$ & $\overline{\text { VVYR }}$ & D & $\overline{\text { GLS }}$ & LPAPVLYA & H & $\overline{L A A K}$ & $\overline{P I W I}$ \\
\hline CePRG-1 & TMIVGY & D & $\mathrm{L}$ & ILYR & D & GAG & VPAPCQYA & H & KLAF & PIWI \\
\hline CePRG-2 & TMIVGY & D & $\mathrm{L}$ & ILYR & D & GAG & VPAPCQYA & H & KLAF & PIWI \\
\hline CeRde-1 & TMYVGI & D & $\bar{V}$ & VVYR & D & GVS & LPVPVHYA & $\mathrm{H}$ & LSCE & III \\
\hline CeF20D12.1 & TFVIGM & D & V & IIFR & D & GVS & IPTPVYVA & H & ELAK & III \\
\hline CeC04F12.1 & TLIISY & D & $\mathrm{V}$ & VILR & D & GVS & LPESIYAA & D & EYAK & $\operatorname{III}$ \\
\hline CeM03D4.6 & LLLIGL & S & $\mathrm{T}$ & VIYL & C & GMS & LPAPLYLT & A & EMAE & III \\
\hline CeK12B6.1 & RLIIGF & $E$ & $\mathrm{~T}$ & LIYF & S & GVS & LPIPLHIA & G & TYSE & III \\
\hline CeF56A6.1 & RLIVGF & V & $\mathrm{T}$ & LLYF & $\mathrm{N}$ & GVS & LPVPLYIA & D & RYSQ & III \\
\hline CePpw-1 & RLIVGF & $\mathrm{V}$ & $\mathrm{T}$ & LLYF & $\mathrm{N}$ & GVS & LPVPLYIA & D & RYSQ & III \\
\hline CeZK1248.7 & QLIIGV & G & $\mathrm{V}$ & IIYR & $\mathrm{S}$ & GAS & IPTPLYVA & $\mathrm{N}$ & EYAK & III \\
\hline CeF58G1.1 & HLIIGV & G & I & IVYR & $\mathrm{T}$ & GTS & LPTPLYVA & $\mathrm{N}$ & EYAK & III \\
\hline CeC06A1.4 & HLIIGV & G & I & IIYR & $\mathrm{T}$ & ETS & LPTPLYVA & $\mathrm{N}$ & EYAK & III \\
\hline CePpw-2 & HLIIGV & G & I & TIYR & S & GSS & IPTPLYVA & $\mathrm{N}$ & EYAK & III \\
\hline CeF55A12.1 & QLIIGV & G & $\mathrm{V}$ & IIYR & S & GAS & IPTPLYVA & $\mathrm{N}$ & EYAK & III \\
\hline CeR06C7.1 & QLIIGV & G & $\mathrm{V}$ & IIYR & $\mathrm{S}$ & GAS & IPTPLYVA & $\mathrm{N}$ & EYAK & III \\
\hline CeY49F6A.1 & TQFIGF & $E$ & $\mathrm{M}$ & VIYR & $\mathrm{T}$ & GAG & VPHILYAA & $\mathrm{D}$ & NLAK & III \\
\hline CeR04A9.2 & TQFIGF & $E$ & M & VVYR & $\mathrm{V}$ & GSG & IPNVSYAA & $Q$ & NLAK & III \\
\hline СeT22H9.3 & VQFIGF & D & I & VIYR & I & GAG & FPDVLYAA & $\mathrm{E}$ & NLAK & III \\
\hline CeC16C10.3 & VQFIGF & $E$ & I & VIYR & $\mathrm{V}$ & GAG & VPDVLYAA & $\mathrm{E}$ & NLAK & III \\
\hline CeC14B1.7 & VQFIGF & $E$ & I & VIYR & V & GAG & VPDVLYAA & $\mathrm{E}$ & NLAK & III \\
\hline
\end{tabular}

Critical aspartates are colored in red, and histidines are colored in dark blue. Substitutions for the DDH motif such as aspartate to glutamate or histidine to either aspartate or glutamate are colored in light red. Substitution of the histidine to a lysine is colored in light blue. 
possibility, we produced hAgo 2 in an organism that lacks the RNAi pathway, namely Escherichia coli (Rivas et al. 2005). This GST-fusion protein was capable of binding siRNA. More importantly, this bacterially expressed protein, programmed with an siRNA, is active for slicing (Rivas et al. 2005). The cleavage position is dependent on the siRNA sequence and the presence of $\mathrm{Mg}^{2+}$ ions. This implies that all that is needed for the slicing reaction is an Argonaute protein and an siRNA, and we therefore called it minimal RISC.

Minimal RISC now enables us to specifically examine what features of the effector step of RNAi reside in Argonaute in combination with the siRNA and what features must come from other components of RISC or perhaps other steps in the pathway. One such feature is the importance of the $5^{\prime}$ phosphate of the siRNA for the slicing reaction itself. We have found that this phosphate is not required for slicing, but that it stabilizes minimal RISC and its presence produces more active enzyme (Rivas et al. 2005). Interestingly, this $5^{\prime}$ phosphate also conveys fidelity to the position of the endonucleolytic cut.

Single-turnover kinetics for the slicing reaction of minimal RISC are remarkably similar compared with holoRISC from fly or human cells (Rivas et al. 2005). However, from previous studies, we know that RISC from Drosophila cell extracts exhibits ATP-stimulated product release during cycling (Nykanen et al. 2001). In the case of minimal RISC, consisting of Argonaute and an siRNA alone, there is no such dependence on ATP (Rivas et al. 2005). This is not surprising, given that there is no indication for an ATP-binding motif in the structure of Argonaute. This further implies that another protein in RISC probably assists in product release using ATP.

Although one can model a duplex RNA in the large groove along PfAgo, the fit is snug. Thus, ATP-stimulated substrate loading and turnover may be dependent on a hinge movement that could open the groove to enable product release and subsequent substrate binding, and close the groove for catalysis. Consistent with this idea, normal mode analysis reveals such a hinge movement to open and close the large groove as well as opening and closing of the smaller groove of PfAgo (Ming et al. 2006). This is also consistent with the rotation of the PAZ domain in AaAgo compared to its position in PfAgo (Yuan et al. 2005).

Nucleotide stimulation was shown, however, for a purification of human RISC that contained Dicer, TRBP, and Ago2 (Gregory et al. 2005). This complex was capable of pre-miRNA cleavage, guide-strand loading, and multiple rounds of mRNA target cleavage. Importantly, this did not require nucleotide hydrolysis.

\section{UNDERSTANDING SLICING}

At this time, we still do not fully understand what makes Argonaute an active Slicer, apart from the required catalytic residues. What exactly determines whether a particular short RNA will mediate target degradation or translational repression? Is the diversification of the Argonautes a consequence of which small RNA they recruit? Is the conformation of the protein dependent on the substrate it targets, or does the protein conformation dictate its targets? Further detailed structural and biochemical experiments will no doubt shed some light on these outstanding questions.

\section{ACKNOWLEDGMENTS}

I thank Niraj H. Tolia for comments on the manuscript. This work was supported by a grant from the National Institutes of Health (R01-GM072659).

\section{REFERENCES}

Baumberger N. and Baulcombe D.C. 2005. Arabidopsis ARGONAUTE1 is an RNA Slicer that selectively recruits microRNAs and short interfering RNAs. Proc. Natl. Acad. Sci. 102: 11928 .

Caudy A.A., Myers M., Hannon G.J., and Hammond S.M. 2002. Fragile X-related protein and VIG associate with the RNA interference machinery. Genes Dev. 16: 2491.

Caudy A.A., Ketting R.F., Hammond S.M., Denli A.M., Bathoorn A.M., Tops B.B., Silva J.M., Myers M.M., Hannon G.J., and Plasterk R.H. 2003. A micrococcal nuclease homologue in RNAi effector complexes. Nature 425: 411.

Doench J.G. and Sharp P.A. 2004. Specificity of microRNA target selection in translational repression. Genes Dev. 18: 504.

Elbashir S.M., Lendeckel W., and Tuschl T. 2001a. RNA interference is mediated by 21- and 22-nucleotide RNAs. Genes Dev. 15: 188

Elbashir S.M., Martinez J., Patkaniowska A., Lendeckel W., and Tuschl T. 2001b. Functional anatomy of siRNAs for mediating efficient RNAi in Drosophila melanogaster embryo lysate. $E M B O ~ J . ~ 20: 6877$.

Gregory R.I., Chendrimada T.P., Cooch N., and Shiekhattar R. 2005. Human RISC couples microRNA biogenesis and posttranscriptional gene silencing. Cell 123: 631.

Hammond S.M., Bernstein E., Beach D., and Hannon G.J. 2000. An RNA-directed nuclease mediates post-transcriptional gene silencing in Drosophila cells. Nature 404: 293.

Hammond S.M., Boettcher S., Caudy A.A., Kobayashi R., and Hannon G.J. 2001. Argonaute2, a link between genetic and biochemical analyses of RNAi. Science 293: 1146.

Irvine D.V., Zaratiegui M., Tolia N.H., Goto D.B., Chitwood D.H., Vaughn M.W., Joshua-Tor L., and Martienssen R.A. 2006. Argonaute slicing is required for heterochromatic silencing and spreading. Science 313: 1134.

Ishizuka A., Siomi M.C., and Siomi H. 2002. A Drosophila fragile X protein interacts with components of RNAi and ribosomal proteins. Genes Dev. 16: 2497.

Lewis B.P., Shih I.H., Jones-Rhoades M.W., Bartel D.P., and Burge C.B. 2003. Prediction of mammalian microRNA targets. Cell 115: 787.

Lingel A., Simon B., Izaurralde E., and Sattler M. 2003. Structure and nucleic-acid binding of the Drosophila Argonaute 2 PAZ domain. Nature 426: 465.

- 2004. Nucleic acid 3 '-end recognition by the Argonaute2 PAZ domain. Nat. Struct. Mol. Biol. 11: 576.

Liu J., Carmell M.A., Rivas F.V., Marsden C.G., Thomson J.M., Song J.J., Hammond S.M., Joshua-Tor L., and Hannon G.J. 2004. Argonaute 2 is the catalytic engine of mammalian RNAi. Science 305: 1437.

Lovell S., Goryshin I.Y., Reznikoff W.R., and Rayment I. 2002. Two-metal active site binding of a Tn5 transposase synaptic complex. Nat. Struct. Biol. 9: 278.

Ma J.B., Ye K., and Patel D.J. 2004. Structural basis for overhang-specific small interfering RNA recognition by the PAZ domain. Nature 429: 318.

Ma J.B., Yuan Y.R., Meister G., Pei Y., Tuschl T., and Patel D.J. 2005. Structural basis for $5^{\prime}$-end-specific recognition of guide RNA by the A. fulgidus Piwi protein. Nature 434: 666. 
Martinez J., Patkaniowska A., Urlaub H., Luhrmann R., and Tuschl T. 2002. Single-stranded antisense siRNAs guide target RNA cleavage in RNAi. Cell 110: 563.

Meister G., Landthaler M., Patkaniowska A., Dorsett Y., Teng G., and Tuschl T. 2004. Human Argonaute2 mediates RNA cleavage targeted by miRNAs and siRNAs. Mol. Cell 15: 185 .

Ming D., Wall M.E., and Sanbonmatsu K.Y. 2006. Domain motions in Argonaute, the catalytic engine of RNA interference. PLoS Comp. Biol. (in press).

Miyoshi K., Tsukumo H., Nagami T., Siomi H., and Siomi M.C. 2005. Slicer function of Drosophila Argonautes and its involvement in RISC formation. Genes Dev. 19: 2837.

Nowotny M., Gaidamakov S.A., Crouch R.J., and Yang W. 2005. Crystal structures of RNase $\mathrm{H}$ bound to an RNA/DNA hybrid: Substrate specificity and metal-dependent catalysis. Cell 121: 1005.

Nykanen A., Haley B., and Zamore P.D. 2001. ATP requirements and small interfering RNA structure in the RNA interference pathway. Cell 107: 309.

Parker J.S., Roe S.M., and Barford D. 2004. Crystal structure of a PIWI protein suggests mechanisms for siRNA recognition and slicer activity. EMBO J. 23: 4727.

. 2005. Structural insights into mRNA recognition from a PIWI domain-siRNA guide complex. Nature 434: 663.

Qi Y., Denli A.M., and Hannon G.J. 2005. Biochemical specialization within Arabidopsis RNA silencing pathways. Mol. Cell 19: 421.

Qi Y., He X., Wang X.J., Kohany O., Jurka J., and Hannon G.J. 2006. Distinct catalytic and non-catalytic roles of ARGONAUTE4 in RNA-directed DNA methylation. Nature (in press).

Rand T.A., Petersen S., Du F., and Wang X. 2005. Argonaute2 cleaves the anti-guide strand of siRNA during RISC activation. Cell 123: 621.

Rivas F.V., Tolia N.H., Song J.J., Aragon J.P., Liu J., Hannon G.J., and Joshua-Tor L. 2005. Purified Argonaute2 and an siRNA form recombinant human RISC. Nat. Struct. Mol. Biol. 12: 340 .

Saito K., Nishida K.M., Mori T., Kawamura Y., Miyoshi K., Nagami T., Siomi H., and Siomi M.C. 2006. Specific association of Piwi with rasiRNAs derived from retrotransposon and heterochromatic regions in the Drosophila genome.
Genes Dev. 20: 2214.

Song J.J., Smith S.K., Hannon G.J., and Joshua-Tor L. 2004. Crystal structure of Argonaute and its implications for RISC slicer activity. Science 305: 1434.

Song J.J., Liu J., Tolia N.H., Schneiderman J., Smith S.K., Martienssen R.A., Hannon G.J., and Joshua-Tor L. 2003. The crystal structure of the Argonaute2 PAZ domain reveals an RNA binding motif in RNAi effector complexes. Nat. Struct. Biol. 10: 1026.

Stark A., Brennecke J., Russell R.B., and Cohen S.M. 2003. Identification of Drosophila MicroRNA targets. PLoS Biol. 1: E60.

Tabara H., Sarkissian M., Kelly W.G., Fleenor J., Grishok A., Timmons L., Fire A., and Mello C.C. 1999. The rde-1 gene, RNA interference, and transposon silencing in C. elegans. Cell 99: 123.

Tijsterman M., Okihara K.L., Thijssen K., and Plasterk R.H. 2002. PPW-1, a PAZ/PIWI protein required for efficient germline RNAi, is defective in a natural isolate of C. elegans. Curr. Biol. 12: 1535 .

Vastenhouw N.L., Fischer S.E., Robert V.J., Thijssen K.L., Fraser A.G., Kamath R.S., Ahringer J., and Plasterk R.H. 2003. A genome-wide screen identifies 27 genes involved in transposon silencing in C. elegans. Curr. Biol. 13: 1311.

Verdel A., Jia S., Gerber S., Sugiyama T., Gygi S., Grewal S.I., and Moazed D. 2004. RNAi-mediated targeting of heterochromatin by the RITS complex. Science 303: 672.

Yan K.S., Yan S., Farooq A., Han A., Zeng L., and Zhou M.M. 2003. Structure and conserved RNA binding of the PAZ domain. Nature 426: 468.

Yigit E., Batista P.J., Bei Y., Pang K.M., Chen C.-C.G., Tolia N.H., Joshua-Tor L., Mitani S., Simard M.J., and Mello C.C. 2006. Analysis of the C. elegans Argonaute family reveals that distinct Argonautes act sequentially during RNAi. Cell (in press).

Yuan Y.R., Pei Y., Ma J.B., Kuryavyi V., Zhadina M., Meister G., Chen H.Y., Dauter Z., Tuschl T., and Patel D.J. 2005. Crystal structure of $A$. aeolicus argonaute, a site-specific DNA-guided endoribonuclease, provides insights into RISCmediated mRNA cleavage. Mol. Cell 19: 405.

Zamore P.D. and Haley B. 2005. Ribo-gnome: The big world of small RNAs. Science 309: 1519. 


\section{$8_{\mathrm{CSH}}^{\infty} \mathrm{H}$ Cold Spring Harbor Symposia SYMPOSIA}

\section{The Argonautes}

L. JOSHUA-TOR

Cold Spring Harb Symp Quant Biol 2006 71: 67-72

Access the most recent version at doi:10.1101/sqb.2006.71.048

References This article cites 39 articles, 15 of which can be accessed free at: http://symposium.cshlp.org/content/71/67.full.html\#ref-list-1

\section{License}

Email Alerting Receive free email alerts when new articles cite this article - sign up in Service the box at the top right corner of the article or click here. 